\title{
MANAGEMENT OF ENDOTHELIAL CORNEAL DYSTROPHY WITH ACRYLIC CORNEAL INLAYS*
}

\author{
BY \\ PETER CHOYCE \\ Southend General Hospital, Essex
}

ENDOTHELIAL corneal dystrophies have always been with us, but that variety which is associated with aphakia seems to be on the increase. This may be an expression of the increasing life-expectation in many societies, so that a higher percentage of the population reaches the cataract age. Recent advances in instrumentation and surgical techniques, e.g., the use of alpha-chymotrypsin, plus improvements in anaesthesia for ophthalmic operations, permit the earlier extraction of immature cataracts and have led to an increase in the number of aphakics.

There is no doubt that aphakic endothelial corneal dystrophy has to be accepted as a disappointment which will occur in a certain percentage of cases. Catford (1964), in an admirable review of the subject, rightly drew attention to the danger of this dystrophy complicating difficulties at the time of cataract extraction, especially if the eye had previously undergone surgery for glaucoma. But it may occur following an uncomplicated extraction in an otherwise healthy eye in a well-behaved patient.

The pathology consists of a progressive and irreversible degeneration in the endothelial lining of the anterior chamber which permits the passage of aqueous through it into the substantia propria of the cornea. At first this oedema is restrained by Bowman's membrane, but later this becomes permeable and the surface epithelium is lifted up in the form of bullae. These burst and re-form. The patient may notice transient improvements in vision following the bursting of a central bulla, usually accompanied by pain due to exposure of corneal nerve endings. This stage of the disease is a great trial to the patient, with recurrent attacks of severe pain, lacrimation, and photophobia, associated with variable but severe diminution of vision. Glaucoma is often a late accompaniment. The water-logged cornea becomes thicker and more opaque, and with lowered resistance is subject to bacterial and viral infections which may administer the coup de grâce to a badly damaged eye.

A common cause is severe post-operative hyphaema, even if the actual extraction proceeds smoothly. The haemorrhage will absorb and the secondary rise of intraocular pressure will settle, but very often some months later the damaged endothelium, particularly in an elderly patient, may become dystrophic, setting in train the sequence of events described above.

It has been stated elsewhere that aphakia plus an anterior chamber implant leads to a slight but perceptible increase in the number of cases of endothelial corneal dystrophy (Choyce, 1964 a). 


\section{Procedures Already Tried}

\section{Techniques}

Local instillations of hypertonic solutions, or ointments to reduce the oedema, or the use of lubricant drops and medicaments achieve nothing. Partial keratectomy and free conjunctival grafting have been recommended; Gundersen $(1958,1960)$ introduced a new technique in the use of conjunctival flaps which Sugar (1964) found efficacious in the relief of pain and irritative symptoms in bullous keratopathy. Tarsorrhaphy offers some relief, but many of these eyes eventually have to be enucleated.

The ideal solution would be to replace the diseased lining of the anterior chamber with healthy endothelium. Total perforating keratoplasty is not feasible and partial perforating keratoplasty in this type of case is disappointing. Occasionally the preliminary insertion of an anterior chamber implant, followed by a partial perforating keratoplasty gives better results (Choyce, 1960; Casey, 1962). Lamellar keratoplasty does not get to the source of the problem, but occasionally a deep lamellar keratoplasty of 6,7 , or $8 \mathrm{~mm}$. diameter seems to help, especially if there is an appreciable junction zone between host and donor cornea, which may hinder the forward passage of aqueous.

Epstein (1964) recommends a $1.5 \mathrm{~mm}$. trephine into the anterior chamber over the site of maximum aqueous seepage. A fornix-based conjunctival flap is carefully sutured to the cut edge of the cornea.

\section{Present Technique}

Since replacement of diseased by normal endothelium is not practicable on a sufficiently large scale in this disease, it was felt that the interposition of a mechanical barrier to the forward movement of aqueous might be beneficial. During 1954 and 1955, while working at the Institute of Ophthalmology, London, I was studying the tolerance of the rabbit and cat corneae to various transparent materials. Glass was not satisfactory, but substantial inlays made of perspex CQ were well tolerated in many instances by the corneae of cats and rabbits, up to the point where some animals died of old age.

The technique used in those experiments has been adapted to the problem of endothelial corneal dystrophy in man. Briefly, the cornea was incised just on the corneal side of the limbus down to a plane of cleavage which exists immediately superficial to Descemet's membrane. A pocket was dissected in this plane of cleavage, into which was inserted a $6 \mathrm{~mm}$. $\times 0.3 \mathrm{~mm}$. disc of perspex CQ with a radius of curvature similar to that of the recipient cornea. Left alone, the acrylic inlays were well tolerated in the majority of the animals, but if the overlying cornea was trephined out an intractable ulcer tended to form. If it was less than $2 \mathrm{~mm}$. in diameter, it would heal, but over $3 \mathrm{~mm}$. in diameter its edges would extend until the inlay was extruded. On the other hand, it was noted that provided the overlying cornea remained intact, extensive laceration of the endothelium and Descemet's membrane from behind, caused by inserting a Ziegler's knife at the limbus, tended to heal with a minimal opacity without disturbing the inlay.

For various reasons this work was never published, but there seems no reason why I should not describe it now, briefly, to lay the foundations of the present technique, which is as follows.

Technique for Insertion of Acrylic Corneal Inlay (Fig. 1, A-E).-It is advisable to practise the technique on the cadaver eye before attempting the operation on a living patient. Under general anaesthesia (but without intravenous acetazolamide (Diamox), which we use routinely for most intra-ocular procedures) the eye is exposed by means of speculum and superior rectus bridle suture. Following preliminary instillation of guttae adrenaline 1 in 1,000 , the superficial capillary plexus from 3 to 9 o'clock is touched lightly with the 


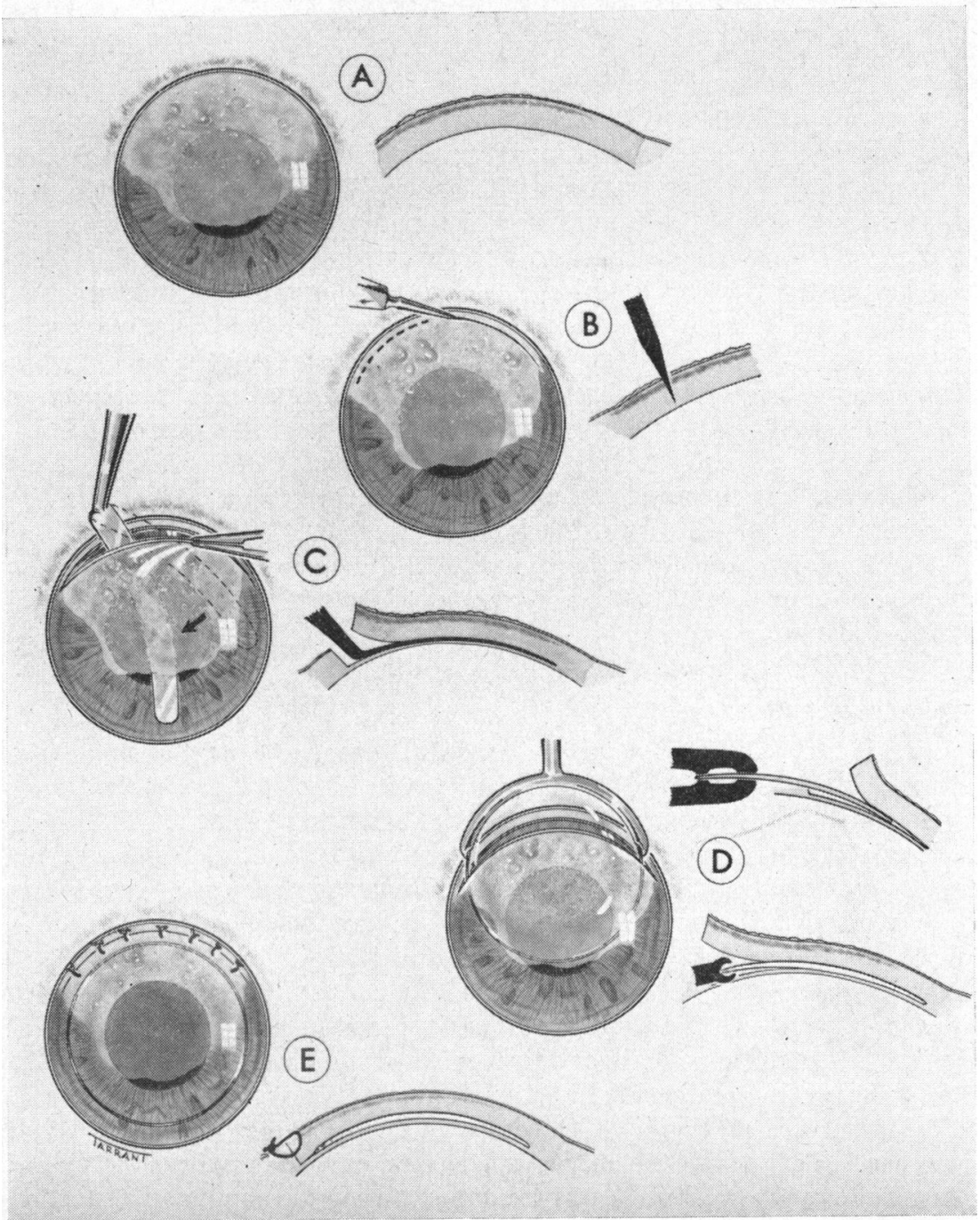

FIG. 1.-Illustrating steps in the technique of inserting acrylic corneal inlays. A, Superficial capillary plexus touched lightly with cautery. B, Cornea incised vertically with Tooke knife. C, Dissection halted about $1 \mathrm{~mm}$. short of the inferior limbus. D, Inlay inserted into pocket. E, Incision closed with interrupted sutures.

cautery (Fig. 1A). If the cornea is covered with thickened, oedematous pannus this tissue should be dissected off Bowman's membrane so that the surgeon can identify the limbus accurately. The cornea is then incised vertically $0.5 \mathrm{~mm}$. on the corneal side of the limbus with a Tooke knife from 9.30 to 2:30 o'clock (Fig. 1B). This is the most anxious part of the operation because while it is necessary to get reasonably close to Descemet's membrane at this stage it is undesirable to open the anterior chamber and lose aqueous humour. Should this occur the operation should not be continued. Assuming that it does not occur (and with practice there should be no aqueous escape) the superficial part of the incised cornea should be grasped with fine Barraquer's corneal forceps, and the plane of cleavage which exists superficial to Descemet's membrane more clearly defined by careful dissection with the Tooke knife. The surgeon requires some form of magnifying spectacles here, 
and excellent illumination. There may be troublesome oozing from the afore-mentioned capillary plexus in spite of the adrenaline drops and the preliminary cauterization. It is undesirable to carry blood into the depths of the cornea and this oozing must be stopped by further application of the cautery. Once this plane of cleavage has been defined to the surgeon's satisfaction, along the length of the incision, the special corneal dissector (which I have designed) should be used to complete the definition of the pocket into which the acrylic disc should be placed. Generally, once the plane of cleavage has been entered, the dissection is easy, and the instrument passes readily along it until it is held up on reaching a point about $1 \mathrm{~mm}$. short of the limbus (Fig. 1C). Some difficulty may be encountered, however, at areas where the cornea has been perforated in the past. If Descemet's membrane is torn before dissection of the pocket is completed, the dissection should be discontinued and the incision sutured, without inserting the inlay. If the cornea is particularly thin or soft and degenerate the dissector may incise the superficial layers. If this buttonhole is no more than $2-3 \mathrm{~mm}$. in extent it will heal, so the inlay should be inserted and the operation completed.

Assuming that this plane of cleavage has been adequately defined and the pocket dissected to the surgeon's satisfaction, any traces of blood which have obtained entry to the pocket should be washed away by gentle irrigation with sterile normal saline at $37^{\circ} \mathrm{C}$. The perspex inlay measures $8 \mathrm{~mm}$. in diameter by $0.2 \mathrm{~mm}$. in thickness. Its radii of curvature are comparable with those of the cornea itself, $7.7 \mathrm{~mm}$. for the anterior radius and $7.4 \mathrm{~mm}$. for the posterior, so designed as to have an afocal effect. Two inlays, sterilized by the caustic soda method of Ridley (1957), are provided by the manufacturers for each case. One inlay is now lifted from its sterile container with Arruga's capsule forceps and inserted into the pocket as far as it will go (Fig. 1D). Sometimes it goes straight in and takes up its proper position without further manipulation; more often it is necessary to manœuvre it so that it is properly centred by using the special pushing device (also designed by me), which has been found of value (Fig. 1D). Having inserted the inlay it only remains to suture the incision; a continuous suture was tried and found unsatisfactory; 5, 6, or 7 interrupted sutures are used (Fig. 1E). Mersuture FM 817 is the most satisfactory; this is an eyeless needle suture of $7 / 0$ braided silk mounted on a reverse cutting double needle, $8 \mathrm{~mm}$. in diameter. These sutures are a pleasure to insert and a joy to remove. (I am obliged to my Registrar, Dr. M. W. Nafie, for suggesting that this type of suture be tried.) Mydriatic drops are instilled and an antibiotic ointment introduced into the conjunctival sac; the eye is padded and the operation is concluded.

Post-operative Care.-At the first dressing on the day after operation the surgeon may be disconcerted by the oedematous aspect of the cornea, but this rapidly clears over the next few days. Acetazolamide (Diamox) $250 \mathrm{mg}$. twice a day assists the process. Homatropine $2 \frac{1}{2}$ per cent. drops are instilled and Betnesol- $\mathrm{N}$ ointment introduced into the conjunctival sac twice daily for 4 to 6 weeks. The patient is sent home after 6 days and the sutures removed between 10 and 14 days after the operation. There have been no complications in this series to date.

\section{Results}

Insertion of an acrylic corneal inlay has been attempted in 34 cases. The first operation was performed in January, 1963. In 32 patients the operation was brought to a satisfactory conclusion, in spite of slight aqueous leakage towards the end of the dissection in 6 cases. In one case with a particularly thin cornea, loss of aqueous at the start of the corneal dissection led to the abandonment of the planned procedure, and the incision was sutured. A modified Cardona type of acrylic prosthesis 
was later inserted into this cornea (Cardona, 1962). The remaining case was found at operation to have a very soft and degenerate substantia propria and it was decided that it would be better to treat this by lamellar keratectomy. The cornea eventually epithelialized quite well and the eye is now painless, but the vision is limited to handmovements only.

The 32 cases in which the operation was properly completed were as follows: (1) Twelve cases of aphakic corneal dystrophy. (2) Nineteen cases of dystrophy occurring in aphakics, plus an anterior chamber implant. Of these, 4 were caused by trauma pushing the implant out of position and up against the cornea; the remaining 15 may be described as idiopathic corneal dystrophies (Choyce, 1964 a). It is interesting to note that the average age of these patients with aphakic dystrophies was 75 years. Clearly an ageing aphakic eye has a particular susceptibility to endothelial degeneration, anterior chamber implant or no anterior chamber implant. (3) One phakic case of Fuchs's dystrophy.

Symptomatic Relief.-It is pleasing to record that the pain and irritability due to bullous keratopathy have been relieved in every case in which they were present. In those cases where it was present in a minor but increasing degree it has regressed. It is interesting to note that the peripheral part of the cornea, outside the circumference of the inlay, still demonstrates small collections of fluid droplets in the subepithelial region, but actual bullae have not formed. The patients have been so thankful to be rid of their recurrent pain and irritability that they have not commented on the failure of this procedure to restore 6/6 vision. In most cases other factors had aIready lowered the expectation of $6 / 6$ vision, so the following statement of the resulting visual acuities may yet be regarded as a modest success. They are: $6 / 24$ in 2 cases; $6 / 36$ in 3 cases; $6 / 60$ in 10 cases; $3 / 60$ in 10 cases; counting fingers at $2 \mathrm{ft}$. in 6 cases; and hand movements in 1 case.

These acuities are usually achieved with a spectacle correction. When the superior corneal incision has healed it seems that the eye becomes more hypermetropic than previously. Corneal sensation is depressed but not abolished following this procedure.

\section{Case Reports}

The points made in this paper may be illustrated by reference to the following cases.

Case 17.-A woman aged 62 years underwent bilateral intracapsular cataract extraction in June, 1963. On the second post-operative night following extraction of the left cataract she slept badly and struck herself in the eye, which filled with blood. There was a secondary rise of tension which lasted for about 72 hours, and it was a fortnight before the hyphaema had completely cleared. Marked striate keratitis remained but gradually lessened until five months after the operation she could see 6/12 with an aphakic correction. Three months later, quite suddenly, the condition of the eye deteriorated (Fig. 2). Endothelial corneal dystrophy spread down from the region of the section to involve the central part of the cornea, with formation of bullae in its upper part. The vision rapidly deteriorated to perception of hand-movements. It is interesting to note that the contralateral undamaged eye exhibits well-marked guttate changes in the endothelium, usually regarded as the precursor of endothelial corneal dystrophy (Fig. 3).

In March, 1964, an $8 \mathrm{~mm}$. $\times 0.2 \mathrm{~mm}$. acrylic corneal inlay was inserted, with immediate improvement in the appearance of the eye. Twelve months later this improvement has been maintained (Fig. 4) and the vision is $6 / 24$ with $+20 \mathrm{DS}$, an increase of 5 dioptres in the pre-dystrophy refraction. 
FIG. 2.-Corneal dystrophy in the left eye of a 62-year-old woman after bilateral intracapsular cataract extraction. Postoperatively she sustained a blow to this eye.
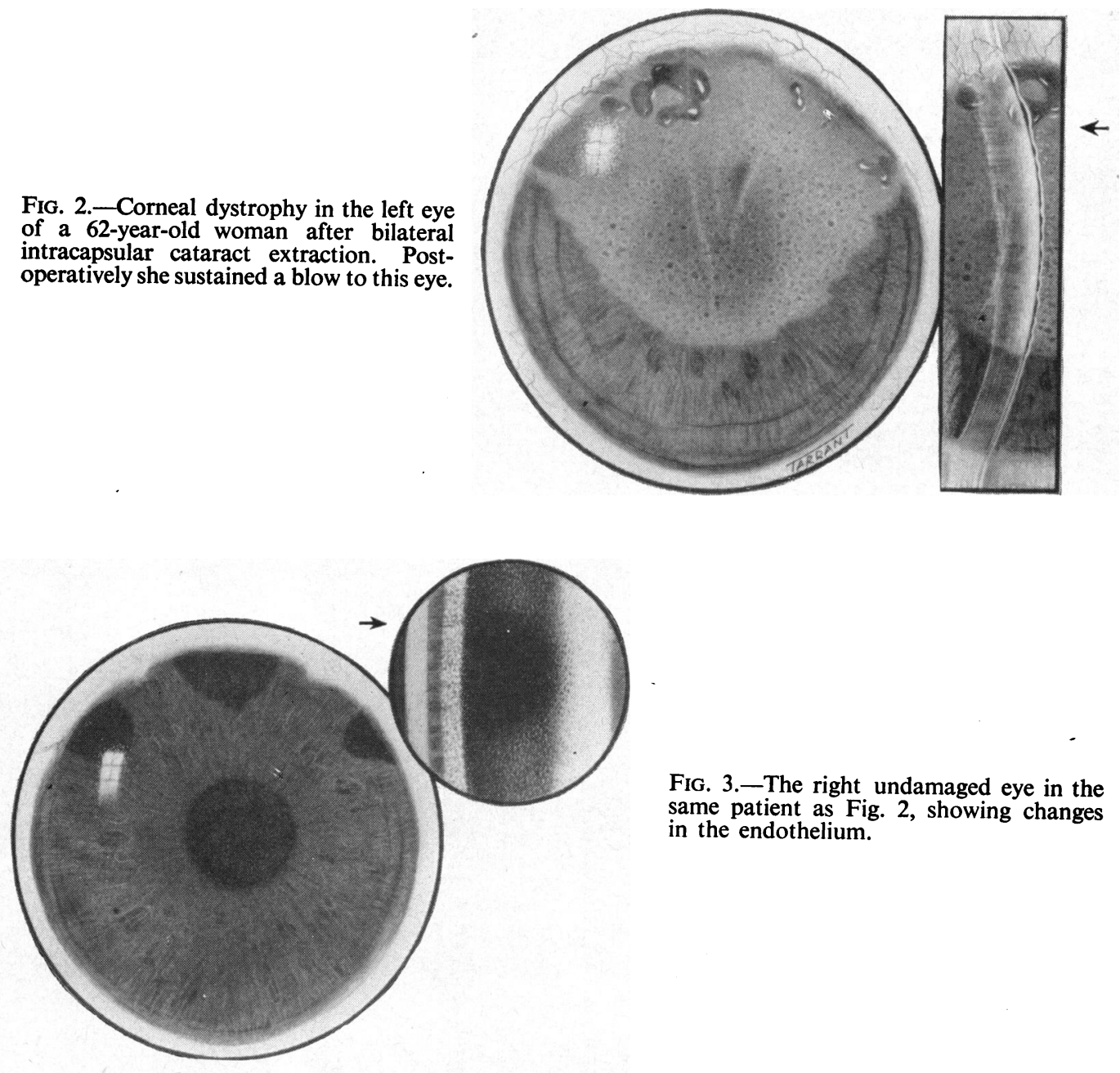

FIG. 3.-The right undamaged eye in the same patient as Fig. 2, showing changes in the endothelium.

Fig. 4.-Left eye of same patient six months after insertion of an acrylic corneal inlay.

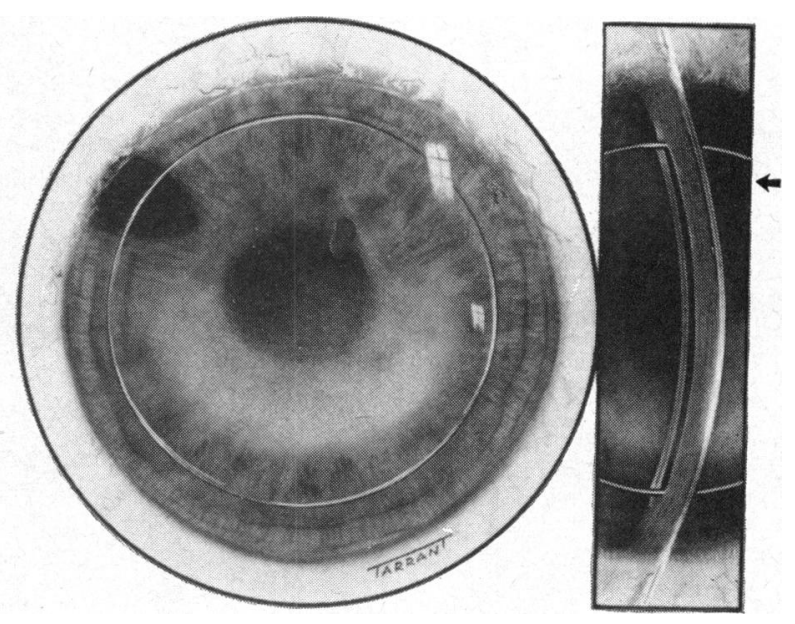


Case 10. - A man aged 70 years had a left intracapsular extraction in May, 1961. In November, 1961, a Choyce Mark V anterior chamber implant was inserted. Three months later the left vision was $6 / 9$ with $-2.5 \mathrm{DC}$ axis 180 . The residual anterior chamber depth was only $1.6 \mathrm{~mm}$., ominously shallow. In December, 1963, endothelial corneal dystrophy developed with formation of bullae in the lower part of the cornea. The vision declined to counting fingers, and there was the usual discomfort associated with bullous keratopathy. In January, 1964, an acrylic corneal inlay was inserted and 18 months later the vision was $6 / 36$ with $+1 \mathrm{D} /-4 \mathrm{DC}$ axis 60 . The eye is white and comfortable and the patient extremely satisfied, although the present visual acuity (6/36) is less than the previous acuity of $6 / 9$.

Case 22.-Bilateral Fuchs's dystrophy of familial origin. At the age of 59 years bilateral $7 \mathrm{~mm}$. lamellar keratoplasty was performed. The operation on the right eye was and is entirely successful, that on the left for some reason was not. Indeed, the condition of the left cornea slowly deteriorated, but instead of regrafting it to relieve what had become well-marked bullous keratopathy, it was decided to insert an $8 \mathrm{~mm}$. acrylic corneal inlay. This was carried out some months ago; the bullous keratopathy has regressed, the eye is much more comfortable, and the vision has improved from hand-movements to $6 / 60$.

\section{Discussion}

Dissecting the cornea in the manner described, and inserting an $8 \mathrm{~mm} . \times 0.2 \mathrm{~mm}$. acrylic corneal inlay, is the most satisfactory means we have yet tried to control the consequences of endothelial corneal dystrophy. Symptomatic relief has been obtained in all cases, and the improvement in visual acuity, though modest, has been welcomed by many patients. As already stated, the problem of aphakic dystrophy of this type seems to be on the increase and this technique is therefore welcome. It is particularly welcome also in the problem of post-anterior chamber implant dystrophies, which have usually been regarded as untreatable and progressing inevitably to blindness and often to loss of the eye.

The existence of this technique by no means encourages the indiscriminate insertion of anterior chamber and other implants into aphakic eyes; on the contrary, the search for the perfect implant and for the safest technique should be intensified.

The Choyce Mark VIII anterior chamber implant (Choyce, 1964 b), while faithful to the original principle of the fixed-length Strampelli-type implant, is proving most satisfactory. It has a dovetail at each end, providing four-point fixation; the flattening of all four feet up to the beginning of the optic portion results in a residual anterior chamber depth approaching $3.0 \mathrm{~mm}$., and the use of an inferior ledge is no longer necessary. Cases of dystrophy associated with the use of any type of implant will always arise, because of the susceptibility of the elderly aphakic eye to just such a degenerative process, with or without the presence of an indwelling implant. It has also been shown that removal of an implant does not prevent the progress of a dystrophy in an aphakic eye once it is firmly established. Indeed, its removal may stimulate progress of the dystrophy by permitting forward movement of the vitreous into the anterior chamber and into contact with the cornea. It is therefore better to insert an acrylic corneal inlay than to remove an anterior chamber implant when confronted with this problem.

The possibility of using this technique in clear corneae is also being investigated. Some success has been achieved in the correction of aphakia by inserting an implant 
which alters the radius of curvature of the cornea. Krwawicz (1960) has used this technique experimentally in rabbits and later in 8 human cases (Krwawicz, 1961), the sequence of events being (1) insertion of acrylic corneal inlay; (2) removal of acrylic corneal inlay after 8-10 days; (3) extraction of cataract after a further 10-14 days.

We have one patient with uniocular aphakia with paralysis agitans, which contraindicated the use of a contact lens; the anterior chamber angle was 60 per cent. occluded, thus contra-indicating the use of an anterior chamber implant. The corneal curvature has been altered in this fashion. Twelve months later the cornea is clear and the visual acuity 6/24, with the inlay still in situ.

These inlays can be made with coloured and opaque portions as with anterior chamber implants (Choyce, 1960). Several blind eyes with clear corneae and ugly iridolenticular remains have received corneal inlays with a $4 \mathrm{~mm}$. coloured haptic surrounding a $4 \mathrm{~mm}$. black optic.

Krwawicz (1960) noted vascular infiltration, necrosis, and even disintegration of the overlying cornea in some of his rabbits observed from 100 to 169 days after implantation of his biconvex inlay. The only late complication in our human series, over the period of 29 months during which this technique has been on trial, has been a tendency to opacification of the superficial part of the cornea where the original dissection was a little less deep than really desirable. This loss of translucency is hinted at in Fig. 4.

\section{Summary}

(1) A technique is described which consists of a deep dissection of the cornea from above, inserting inlays of perspex CQ into the pocket so fashioned.

(2) By acting as a mechanical barrier to forward movement of aqueous through damaged endothelium and Descemet's membrane, bullous keratopathy has been caused to regress, and there has been a modest restoration of visual acuity, in a series of 32 patients operated upon over the last 21 months.*

(3) The procedure is equally effective in ordinary aphakia and in aphakia plus an anterior chamber implant.

(4) This technique may have a cosmetic application as an alternative to a cosmetic contact lens or anterior chamber implant, and may also be used to correct uniocular aphakia if the cornea is clear by using an inlay which increases the corneal curvature.

I thank Sir Stewart Duke-Elder, Director of Research, Institute of Ophthalmology, for encouragement with the animal experimentation ten years ago, from which this technique is derived.

The inlays have been made and sterilized by Rayners Ltd., and the figures prepared by Mr. T. R. Tarrant; I am most grateful for their help. Imperial Chemical Industries Ltd. are the sole manufacturers of perspex CQ. Down Bros. Ltd. made the special corneal dissector and inlay introducer.

* A further 11 inlays have been inserted during the last eight months, confirming the results recorded here. 


\section{REFERENCES}

Cardona, H. (1962). Amer. J. Ophthal., 54, 284.

CASEY, T. A. (1962). "International Ophthalmology Clinics", ed. P. D. Trevor-Roper, vol. 2, no. 3, p. 801. Little, Brown, Boston.

CATford, G. V. (1964). Trans. Ophthal. Soc. U.K., 76, 35.

CHOYCE, D. P. (1960). Ibid., 80, 201. (1964 a). "Intra-ocular Lenses and Implants", chap. 14, p. 103. H. K. Lewis, London. (1964 b). J. Soc. Ciênca Med. Lisboa, 128, 665.

EPSTEIN, E. (1964). Personal communication.

Gundersen, T. (1958). A.M.A. Arch. Ophthal., 60, 880. (1960). Ibid., 64, 260.

Krwawicz, T. (1960). Klin. oczna, 30, 229. (1961). Brit. J. Ophthal., 45, 59.

RidLEY, F. (1957). Ibid., 41, 359.

Sugar, H. S. (1964). Amer. J. Ophthal., 57, 977. 